\title{
Inaccurate Flow Rate
}

National Cancer Institute

\section{Source}

National Cancer Institute. Inaccurate Flow Rate. NCI Thesaurus. Code C63102.

Problem associated with fluctuations in the flow volume delivered per time, even if end volume is correct, and delivered in the correct total time. 